\title{
Uso de la Educación Virtual en los postgrados de la Facultad de Humanidades y Ciencias Jurídicas de la UNAN-Managua
}

\author{
Ángela López Tórrez \\ Responsable TIC Fac. Humanidades y C.J. - Universidad Nacional Autónoma de Nicaragua, Managua \\ alopez@unan.edu.ni \\ Ledyth López Herández \\ Docente Universidad Nacional Autónoma de Nicaragua, Managua \\ ledythlafilologa@yahoo.es
}

Fecha de recibido: 6 de abril de 2012

Fecha de aprobado: 12 de septiembre de

2015

\section{RESUMEN}

Debido al avance tecnológico en el campo de la educación, se hace estrictamente necesario, que los estudiantes de postgrado de la Facultad de Humanidades y Ciencias Jurídicas, tengan un acercamiento adecuado al uso de las tecnologías de entornos virtuales de aprendizaje, las cuales constituyen un aporte muy significativo para el mejoramiento de la calidad educativa. En la actualidad se enfrentan muchas limitantes en cuanto al uso de estos recursos tecnológicos educativos y se está trabajando en miras del fortalecimiento del área de informática y en la elaboración de planes de capacitaciones para el personal docente y estudiantes, con el fin de ir superando paulatinamente estas debilidades y lograr un mayor desarrollo en la virtualización de la educación en los estudios de postgrado. En vista de lo anterior, la Facultad considera como prioritario la implementar de un modelo de capacitación que posteriormente se aplique tanto a docentes como a estudiantes de los postgrados que se imparten actualmente en la Facultad. Previo a la implementación de estas capacitaciones es recomendable que la Facultad adquiera equipamiento informático y recursos humanos que vengan a fortalecer la Unidad TIC y de esta forma poner a disposición de los participantes de posgrado, los medios y las herramientas necesarias para que puedan tener acceso a estas aplicaciones virtuales y se les pueda brindar el soporte técnico necesaria a los usuarios. Existe seguridad en expresar que el trabajo constante en esta línea, pronto se materializará en un mayor grado de desarrollo en la creación y uso de entornos virtuales de enseñanza aprendizaje.
Palabras claves: TIC, NTIC, Educación, Tecnologías, educación virtual, aulas virtuales, aprendizaje, limitaciones, comunicación

\section{ABSTRACT}

Due to technological advancement in the field of education, it is absolutely necessary that students graduate of the Faculty of Humanities and Legal Sciences, have adequate approach to the use of technologies of virtual learning environments, which constitute a contribution very significant for improving educational quality. At present many constraints faced in the use of these educational technology resources and is working towards strengthening the area of information and the development of training plans for teachers and students to gradually overcome gradually these weaknesses and achieve greater development in the virtualization of education in postgraduate studies. In view of the above, the Faculty considers as a priority the implementation of a training model that is subsequently applied to both teachers and students of the graduate degrees currently offered in the Faculty. Prior to the implementation of this training is recommended that the Faculty acquire computer equipment and human resources to come to strengthen the ICT Unit and thus make available to participants graduate, media and tools so they can have access to these virtual applications and they can provide the necessary support to users. There is safe to say that the constant work in this line, suddenly materialize in a greater degree of development in the creation and use of virtual teaching and learning. 
Hablar de educación virtual implica abordar diferentes temas, entre ellos el cambio que ha tenido la educación con el uso de la tecnología. Así podemos comentar que las Nuevas Tecnologías de la Información y la Comunicación (NTIC) se han convertido en uno de los pilares básicos en la educación superior y los entornos virtuales de aprendizaje formar parte de estas tecnologías educativas.

Es importante destacar, que las nuevas modalidades de enseñanza en relación a las TIC son indispensable en la educación, por lo que se hace necesario tener un acercamiento adecuado a este tipo de herramientas, puesto que favorecen la introducción de aspectos innovadores en la metodología relacionada con los procesos de enseñanza y aprendizaje.

Por ende la utilidad plena de los entornos virtuales de aprendizaje en el contexto educativo de enseñanza en los postgrados de la Facultad de Humanidades y Ciencias Jurídicas, beneficiaría el sistema educativo en su conjunto, facilitando la enseñanza amplia a través de la interacción, retroalimentación y participación colaborativa entre docentes y estudiantes.

Cabe destacar que con la reciente construcción del Centro de Difusión para las Humanidades (CDIHUM), la Facultad de Humanidades y Ciencias Jurídicas, ha obtenido un avance tecnológico muy significativo, debido a que este centro fue creado con el objetivo de difundir el quehacer académico de la Facultad, haciendo uso de la tecnología digital.

Dentro de este edificio fue construido el cuarto de servidores o centro de datos, donde se encuentra instalado el nodo de fibra óptica de la Facultad, con los equipos de redes necesarios para conectividad a Internet.

En esta misma línea, podemos mencionar que en la Facultad, se ha diseñado un sitio web, moderno, dinámico y muy atractivo, a través del cual se mantiene infirmada a la comunidad universitaria y público en general a cerca de las actividades académicas que se realizan constantemente en los diferentes departamentos, carreras y dependencias de la Facultad.

Así mismo, dentro del sitio web, se establece enlace a una plataforma de aprendizaje virtual que está siendo usada por estudiantes y docentes de grado y postgrado, como una herramienta de apoyo a las clases presenciales. El desarrollo de esta herramienta de aprendizaje no se ha podido alcanzar de forma plena, debido a que existen varias limitantes.

Dentro de estas se mencionan las siguientes:

Facultad: 
1. En la actualidad la Facultad dispone de un nodo de redes con un enlace de fibra óptica y equipos de conectividad, pero hace falta el equipamiento (servidores y unidades de almacenamiento) necesario para la implementación de aplicaciones de tecnología educativa.

2. La Facultad no cuenta con equipo técnico destinado a la creación y administración de los cursos virtuales en Posgrado, Diplomados y Doctorados.

3. La Facultad no cuenta con especialista o expertos en la creación y uso de cursos virtuales con pedagogía adecuada, para implementar las capacitaciones.

4. La docencia de posgrado de la Facultad todavía no ha recibido un entrenamiento intensivo en la creación de ambientes virtuales con pedagogía adecuada.

5. En la Facultad el ancho de banda disponible para el acceso a Internet es un tanto insuficiente.

6. El acceso a los laboratorios y a equipos clientes es limitado debido a que en la actualidad solamente existe un laboratorio de computación.

Docente:

1. La falta de capacitación de docentes en el uso de los entornos virtuales de aprendizaje, contribuye a la poca familiarizados con estas tecnologías.

2. Muchos docentes de posgrados están dispuestos a transmitir sus conocimientos utilizando estos recursos, pero no tienen el entrenamiento necesario para hacerlo.

3. En menor grado, se puede decir que existen docentes que demuestran una actitud resistente a estas nuevas modalidades de enseñanza-aprendizaje.

4. Los docentes de esta facultad tienen un menor entrenamiento en el tema de las tutorías en línea, tema que es muy importante para dar seguimiento al avance de los estudiantes.

5. Compañías ofrecen a usuarios individuales precios módicos pero con menor capacidad de ancho de banda, por lo que esto provoca atraso en el acceso a los campus virtuales.

6. Algunos docentes que imparten los posgrados, ven de manera negativa la creación de los cursos virtuales, puesto opinan que les consume un mayor tiempo el proceso de preparación y subida de la información en la plataforma.

Estudiante:

1. Los estudiantes de posgrado carecen de capacitación adecuada, en el uso de estos recursos tecnológicos, problema que se origina desde el pregrado.

2. Debido a que el acceso a internet es costoso, pocos estudiantes de posgrado puedan acceder a los entornos virtuales en sus hogares. 
3. El ancho de bandas con los que cuentan los estudiantes para acceso a internet en sus hogares son bajos, puesto que las tarifas de costo dependen de la cantidad de ancho de banda que soliciten.

4. Por experiencia en los estudios de posgrado, se puede afirmar que no todos los estudiantes cuentan con el equipo adecuado para acceder a las diferentes plataformas virtuales.

Referente a lo mencionado, se recomienda implementarse una estrategia de capacitación a docentes y estudiantes de los postgrados, para crear una cultura de uso permanente de los entornos virtuales de aprendizaje, eliminando así la resistencia al uso de estas herramientas que son de utilidad básica en la enseñanza de educación superior.

Las TIC están produciendo cambios en la formas de enseñanza y aprendizaje desplazando paulatinamente los métodos tradicionales (graduados de folletos), logrando así una mayor gestión de la información, puesto que los docentes incorporan su material didáctico con anticipación, facilitando al alumno la comprensión y análisis de la temática a estudiar, dando pautas a nuevos conocimientos que son complementarios a la enseñanza principal.

Los espacio colaborativa facilitan la retroalimentación, en el caso de las aulas virtuales son de mayor importancia, puesto que sirven de complemento a la enseñanza tradicional haciendo de esta un método inteligente, interactivo y atractivo, lo que le permite al docente lograr que los estudiante aprendan a trabajar en equipo y generar mayores conocimientos.

Dory Araujo de Cendros y José Bermudes, en el artículo publicado en 2009, sobre Limitaciones de las Tecnologías de Información y Comunicación en la Educación Universitaria, refiere que:

Las Tecnologías de Información y Comunicación (TIC) posibilitan la creación de un nuevo espacio social de carácter virtual en la educación. En este nuevo entorno, se está desarrollando la educación, obligando a las instituciones educativas, a plantearse cambios en sus estructuras, para evitar quedar marginados ante el avance tecnológico.

Sustentando esta afirmación podemos decir que la UNAN - Managua forma parte de estos cambios, puesto que es una institución que brinda un servicio público a la comunidad, debido a esto gradualmente la universidad ha asumido el reto de actualizarse en tecnologías educativas, en aras de mejor la calidad de la enseñanza superior y competir a la vez con otras universidades en el área virtual.

Para lograr un mayor nivel de avance tecnológico se deben establecer lineamientos, políticas, normas, estrategias de desarrollo y destinar un presupuesto para la utilidad de estos recursos tecnológicos 
desde la dirección superior de UNAN-Managua, posteriormente implementarlas a través de las diferentes facultades de esta alma mater y sus sedes regionales.

Paralelo, se debe realizar una estrategia permanente a corto, mediano y largo plazo para la ejecución del planteamiento anterior, asimismo debe validarse con los miembros de la dirección superior de esta universidad para medir los niveles de asertividad y aceptación de esta propuesta, que contribuirá a ese inserción de los entornos virtuales desde la educación secundaria (prepa), carreras técnicas, cursos libres y otras modalidades de enseñanza que ofrece la UNAN-Managua.

Todo esto contribuirá a la facilidad del aprendizaje de los postulantes a posgrados, puesto que tendrán un conocimiento básico en relación al uso de estas Nuevas Tecnologías de la Información y la Comunicación.

En el caso de la Facultad de Humanidades y Ciencias Jurídicas se está trabajando en esta línea, para lograr fortalecer la Unidad TIC, tanto en la adquisición de equipamiento informático, infraestructura de redes que sirve de base para la implementación de aplicaciones de tecnología educativa, así como la contratación de recursos humanos necesarios para integrar el equipo de trabajo de esa área y de esta forma brindar un mejor servicio a la comunidad universitaria de la Facultad.

Cabe señalar que esta unidad es la que pone a disposición los medios, herramientas y aplicaciones necesarias para el uso de los entornos virtuales de aprendizaje, donde los docentes pueden subir sus recursos digitales en diferentes formatos y les permiten además participar en redes de docentes multidisciplinarios, aprovechando de esa forma la oportunidades de aumentar sus conocimientos y luego transmitírselos a sus estudiantes.

Así mismo esta unidad es la que se encarga también de atender las incidencias de usuarios, cuando estos tienen problemas con el uso de los recursos tecnológicos que se han puesto a disposición de la comunidad universitaria de la esta facultad, entre los más comunes están:

1. Registro de usuario en la base datos de la plataforma virtual (Creación de cuentas a Docentes y Estudiantes).

2. Cambio de contraseña para los usuarios, que reportan problemas de acceso a la plataforma.

3. Creación de cursos virtuales a solicitud de los docentes.

4. Asistencia técnica a docentes que tienen dificultad al crear recursos y actividades dentro de los cursos virtuales.

5. Asistencia técnica a los docentes, al estructurar el curso. 
6. Asistencia técnica a estudiantes que tienen algún inconveniente en el uso de la plataforma virtual

Solucionados los problemas técnicos, de equipamiento, recursos humanos y de capacitación al personal docente de posgrado, la Facultad de Humanidades y Ciencias Jurídicas puede implementar de manera plena el uso de los entornos virtuales de aprendizaje, cumpliendo así con objetivos y metas planteadas durante los cursos. Todo esto constituye desde el punto de vista académico, un logro determinante debido a que nuestros profesionales podrán estudiar las modalidades de posgrados con mayor profundidad haciendo uso de los entornos virtuales de enseñanza-aprendizaje, siendo ellos al final del curso profesionales competentes, hábiles y propositivos de estas nuevas formas de enseñanzas.

Enfocándonos en el área de Tecnologías de la Información y Comunicación es preciso establecer una cultura de uso permanente de estos recursos, en pro de la educación superior, de las competencias nacionales e internacionales de enseñanza virtual, porque las empresas, ministerios, organizaciones, instituciones del estado, entidades privadas etc., trabajan bajo esa línea de actualización y proyección virtual.

En este mismo ámbito, es necesario implementar métodos de concientización y motivación tanto en la docencia como en el estudiantado, esto debe hacerse de manera constante y paralela al uso de estos recursos, así mismo se debe hacer una campaña de difusión desde el Centro de Difusión de las Humanidades, para lograr la proyección de estos recursos y un cambio de actitud en los docentes que se resisten a la innovación virtual.

Cabe señalar que la tecnología al igual que las ciencia del conocimiento, avanzan a pasos agigantados y el mantenerse al margen del desarrollo tecnológico, crea una brecha entre quienes tienen los medios adecuados para hacer uso de la tecnología y de quienes están un poco excluidos del uso de ésta, como consecuencia se generan limitaciones en el desarrollo de habilidades, destrezas comunicativas y desarrollo de un nuevo potencial en las innovaciones tecnológicas educativas.

Esto lo podemos argumentar con un fragmento de la revista Infobit (2005) que dice:

En el ámbito específico de las TIC, éstas han venido transformando la realidad social, situación que está a la vista, pues han permeado todos los ámbitos de la vida: familiar, el educativo, el laboral, el comunitario, el político y hasta el privado. La ola de despliegue tecnológico, cada vez más sofisticada a partir de finales del siglo XX y comienzos del XXI, ha simplificado muchas actividades que antes eran lentas, engorrosas o de elevados 
costos. El individuo, por su parte, ha podido enriquecer sus saberes sobre la realidad local, regional, nacionales internacional que lo circunda (p. 26)

Por muchos convenios que las universidades, instituciones, organizaciones y empresas suscriban, si no se invierte en una infraestructura adecuada que contemple, tecnología avanzada, y no se capacite de manera sistemática a los recursos humanos para el uso de esas nuevas herramientas de tecnología educativa, las posibilidades de un "aislamiento" del avance científico, tecnológico y de competitividad en el campo laboral y profesional es mayor.

El uso de las plataformas virtuales de enseñanza-aprendizaje, permiten relevar propuestas metodológicas de educación tradicional y modelos pedagógicos obsoletos (graduados de folletos), por lo que se ha tomado en consideración que la Facultad de Humanidades y Ciencias Jurídicas, no se puede quedar atrás ante esta realidad virtual que se está viviendo.

Por otra parte se puede afirmar que la comunicación permite mantener un flujo de información entre el profesor y los participantes, que les facilita: poner en común ideas, compartir, reflexionar, desarrollar trabajos de carácter cooperativo y/o colaborativo, recibir retroalimentación y orientaciones por parte del tutor. En base a lo anterior podemos afirmar que es prioritario implementar una estrategia de capacitación que permita a docentes y estudiantes de los postgrados de la Facultad de Humanidades y Ciencias Jurídicas, tener una base metodológica para ir introduciéndose de lleno al uso de los entornos virtuales de aprendizaje, aunque inicialmente se realicen los cursos de manera semi-presencial, es decir, que la mitad del contenido sea abordado de manera presencial y la otra mitad sea impartido en línea. A futuro se tiene proyectado, implementar los estudios de posgrado de la Facultad, totalmente en línea.

Esto es con el fin de avanzar poco a poco en la inserción de esta metodología de enseñanza aprendizaje virtual, ya que actualmente la mayoría de los docentes presentan una actitud de resistencia ante el cambio de la educación tradicional hacia la educación donde se hace uso de entornos virtuales, esto se da debido al desconocimiento del uso de estas herramientas, otro factor de resistencia es que existen muchos docentes de edad avanzada, quienes demuestran poco o ningún interés en incursionar en el aprendizaje de este tipo de tecnología.

En relación a los estudiantes, las limitaciones del uso de estos recursos tecnológicos se dan primeramente porque en los docentes no se ha desarrollado todavía una cultura de uso permanente de estos recursos tecnológicos, por lo que no existen exigencias de uso por parte de ellos hacia los estudiantes, por otra parte los estudiantes no cuentan con los medios y equipos necesarios para hacer uso 
de esta tecnología educativas cuyo uso es de vital importancia debido a que constituyen un aporte esencial en el mejoramiento de la calidad educativa.

Por muchos convenios que las universidades, instituciones, organizaciones y empresas suscriban, si no se invierte en una infraestructura adecuada que contemple, tecnología de avanzada, y no se capacite de manera sistemática a los recursos humanos para el uso de esas nuevas herramientas de comunicación, las posibilidades de un "aislamiento" del avance científico, tecnológico y de competitividad en el campo laboral es mayor.

Al concluir este análisis podemos darnos cuenta de la importancia que tiene el uso de la tecnología educativa en la comunidad universitaria, por lo que afirmamos que es ahora un reto para la Facultad de Humanidades y Ciencias Jurídicas, la elaboración de un modelo de capacitación y luego la aplicación del mismo, en el uso de entornos virtuales de aprendizaje tanto a nivel de docentes como para estudiantes de los postgrados que se están impartiendo.

Cabe mencionar que previo al cumplimiento con la labor de capacitación, la Facultad de Humanidades y Ciencias Jurídicas debe poner a disposición de los participantes de postgrados, los medios y las herramientas necesarias para que esta actividad se pueda realizar sin contratiempos, pues nos mueve el interés de construir un modelo de educación pedagógica más allá de lo tradicional y esto implica la adquisición de equipamiento informático y recursos humanos que contribuyan al fortalecimiento de la Unidad TIC.

Podemos expresar con propiedad que si la Facultad de Humanidades y Ciencias Jurídicas trabaja de cara al fortalecimiento de la Unidad TIC tanto en equipamiento como en recursos humanos y la implementación de capacitaciones en el uso de entornos virtuales de aprendizaje, muy pronto se logrará el desarrollo en un alto grado en la virtualización de los cursos de postrado, tanto así que estos cursos ya no se impartirían de manera semi-presencial sino en una modalidad totalmente en línea.

Lo anterior, sería un gran avance debido a que los participantes de postgrados tendrían los conocimientos necesarios en el uso de los diferentes recursos que ofrecen los entornos virtuales de aprendizaje y podrían aprovechar al máximo el potencial que ofrece la virtualización.

Al capacitar tanto a los docentes como a los estudiantes en el uso de estas plataformas virtuales, la Facultad no solo lograría el desarrollo de la virtualización de la educación sino que también esto constituiría un gran aporte al mejoramiento de la calidad educativa, debido a que los estudiantes de 
postgrado saldrían con una preparación superior y se convertirían en profesionales con mayor competitividad en el campo laboral.

Para finalizar instamos a la comunidad universitaria a asumir una actitud positiva hacia el desarrollo tecnológico y de esta forma puedan aprender a disfrutar del cambio, innovación y versatilidad que trae consigo el uso de la tecnología.

\section{REFERENCIAS}

Caicedo-Tamayo, A. M., \& Rojas-Ospina, T. (2014). Creencias, conocimientos y usos de las TIC de los profesores universitarios. (Spanish). Educación Y Educadores, 17(3), 517-533. doi:10.5294/edu.2014.17.3.7

Orozco, H. (2013). Claves para una integración equilibrada de los usos de las TIC en el proceso de enseñanza-aprendizaje. (Spanish). Revista Cultura De Guatemala, 34(1), 75-104.URL: http://search.ebscohost.com/login.aspx?direct=true\&db=zbh\&AN=91872940\&lang=es\&site=eho $\underline{\text { st-live }}$

López de la Madrid, M. C. (2007). Uso de las TIC en la educación superior de México. Un estudio de caso. (Spanish). Apertura: Revista De Innovación Educativa, 7(7), 63-81. URL: http://search.ebscohost.com/login.aspx?direct=true \&db=zbh\&AN=28627028\&lang=es\&site=eho $\underline{\text { st-live }}$ 